\title{
Determinación de los mecanismos de fractura de un material multicapa de aluminio de alta resistencia y excelente tenacidad a impacto basado en la aleación aeroespacial Al $7075^{(\bullet)}$
}

\author{
C. M. Cepeda-Jiménez*, O. A. Ruano* y F. Carreño*
}

\begin{abstract}
Resumen
En este trabajo se ha procesado mediante laminación en caliente un material multicapa constituido por 19 capas alternadas de aluminio de alta resistencia ( $\mathrm{Al} 7075-\mathrm{T} 6,82 \% \mathrm{vol}$ ) y capas de aluminio puro (Al 1050-H24, $18 \%$ vol) de menor espesor. Se ha caracterizado la microestructura de las aleaciones constituyentes después del procesado, así como el gradiente de composición generado alrededor de las intercaras. Las propiedades mecánicas a temperatura ambiente, tanto del material multicapa como de las aleaciones de partida, se han estudiado mediante microdureza Vickers, flexión en tres puntos y ensayos de impacto Charpy. El material multicapa procesado presenta una tenacidad a impacto Charpy 18 veces superior a la de la aleación de partida Al 7075. Este espectacular aumento de tolerancia al daño es debido a los mecanismos de fractura, tanto extrínsecos como intrínsecos, que operan en el material multicapa durante las diferentes solicitaciones mecánicas a las que ha sido sometido.
\end{abstract}

Palabras clave Al 7075; Material compuesto multicapa; Intercaras; Procesado termomecánico; Tolerancia al daño.

\section{Fracture mechanisms assessment of a multilayer material with high strength and excellent impact toughness based on the aerospace Al 7075 alloy}

\begin{abstract}
An aluminium multilayer laminate has been processed by hot rolling. It is constituted by 19 alternated layers of high-strength aluminium alloy (Al 7075-T6, $82 \% \mathrm{vol}$ ) and thinner pure aluminium layers ( $\mathrm{Al}$ 1050-H24, $18 \% \mathrm{vol}$ ). The microstructure of the constituent alloys and the composition gradient across the interfaces has been characterized. The multilayer laminate and the as-received aluminium alloys have been tested at room temperature by Vickers microhardness, three-point bend test and impact Charpy test. The outstanding improvement in damage tolerance, which is 18 times higher than that for the as-received $\mathrm{Al} 7075$ alloy, is due to both intrinsic and extrinsic fracture mechanisms operating in the multilayer laminate during mechanical testing.
\end{abstract}

Keywords

Al 7075; Multilayer composite material; Interfaces; Thermomechanical processing; Damage tolerance.

\section{INTRODUCCIÓN}

Los materiales compuestos multicapa han sido objeto de numerosos estudios en los últimos años debido a las notables mejoras que se obtienen en cuanto a propiedades mecánicas, magnéticas o eléctricas ${ }^{[1-5]}$. La utilización de materiales multicapa en estructuras para el transporte aeroespacial está condicionada por su tolerancia al daño ${ }^{[6]}$, por lo que este concepto es de gran importancia en el diseño de materiales para garantizar su integridad estructural ante la posibilidad de cualquier daño.

Mediante laminación en caliente se pueden procesar materiales multicapa, de forma que a través de las condiciones de procesado adecuadas se pueden conseguir mejoras sorprendentes de tenacidad, sin detrimento de la resistencia mecánica del material $^{[7]}$.

De forma general, los factores que influyen en la tenacidad a fractura se pueden clasificar en intrínsecos y extrínsecos. Los mecanismos intrínsecos son inherentes al material (efectos del tamaño de grano, precipitados, distancia entre partículas...). Por el contrario, mecanismos extrínsecos son, por ejemplo, la delaminación en las intercaras. De hecho, la presencia de intercaras es la responsable del aumento de tenacidad observado en materiales multicapa ${ }^{[8-13]}$.

(•) Trabajo recibido el día 02 de febrero de 2012 y aceptado en su forma final el día 13 de abril de 2012.

* Departamento de Metalurgia Física, CENIM-CSIC, Avda. Gregorio del Amo 8, 28040 Madrid. 
Las aleaciones de aluminio de más alta resistencia son las pertenecientes a la serie $7 \mathrm{xxx}, \mathrm{Al}-\mathrm{Zn}-\mathrm{Mg}-\mathrm{Cu}{ }^{[14]}$. Sin embargo, estas aleaciones presentan escasa tenacidad, lo que limita su uso en ciertas aplicaciones. Por tanto, el gran reto es obtener materiales con elevada resistencia y simultáneamente alta tenacidad.

Aunque la industria del aluminio continúa mejorando constantemente las formulaciones de las aleaciones para mejorar propiedades, la gran oportunidad para dar un salto cuantitativo es diseñar nuevas configuraciones de materiales donde se potencien sus puntos fuertes, como por ejemplo la alta resistencia de las aleaciones de aluminio, y se minimicen sus puntos débiles como la baja tenacidad.

En este trabajo se ha procesado mediante laminación en caliente un material multicapa constituido principalmente por capas de la aleación de aluminio de alta resistencia $\mathrm{Al} 7075$, la cual constituye el $82 \%$ vol del material compuesto. Para facilitar la unión entre las capas de aluminio de alta resistencia se han intercalado capas más delgadas de aluminio puro $\mathrm{Al} 1050$, las cuales constituyen el $18 \%$ vol, y que contribuirán también a la tenacidad intrínseca del material multicapa ${ }^{[15]}$. Por tanto, el objetivo de este trabajo es caracterizar la microestructura y las propiedades mecánicas del material multicapa, especialmente desde el punto de vista de la mejora en la tenacidad a impacto y los mecanismos de fractura responsables de dicha mejora.

\section{MÉTODO EXPERIMENTAL}

\subsection{Procesado}

Las aleaciones de aluminio utilizadas para el procesado del material multicapa fueron chapas laminadas de Al 7075 comercial en estado inicial T6 $(5,68 \mathrm{Zn}-2,51 \mathrm{Mg}-1,59 \mathrm{Cu}-0,19 \mathrm{Cr}$ y balance en peso de $\mathrm{Al}$ ) y referenciada en este trabajo con la letra "D", y de Al 1050 en estado inicial $\mathrm{H} 24(0,12 \mathrm{Si}-0,21 \mathrm{Fe})$ y referenciada en este trabajo con la letra "H". Algunas propiedades mecánicas de las aleaciones de partida se muestran en la tabla I. Las dimensiones de las chapas de partida fueron $150 \times 60 \times 2 \mathrm{~mm}^{3}$.

El material multicapa se realizó apilando 10 chapas de $2 \mathrm{~mm}$ de espesor de la aleación Al 7075-T6 (D), alternadas con 9 chapas de $0,5 \mathrm{~mm}$ de espesor de la aleación $\mathrm{Al} 1050(\mathrm{H})$, las cuales fueron previamente laminadas desde el espesor de partida hasta el considerado en este estudio. La relación en volumen Al 7075:Al 1050 fue por lo tanto de 82:18.

Para evitar la entrada de oxígeno y el deslizamiento de las chapas durante el procesado de laminación, el apilamiento de chapas fue soldado a lo largo de todo su perímetro mediante soldadura TIG. Posteriormente, el tocho resultante fue laminado en caliente a $465^{\circ} \mathrm{C}$, temperatura a la cual se produce la solubilización de la aleación Al 7075, mediante varios ciclos de 3-4 pasadas cada uno. En la figura 1 se muestra un esquema de las temperaturas registradas durante todo el proceso de laminación. La reducción de espesor por pasada fue del 4-8 \%, siendo la muestra calentada de nuevo a $465^{\circ} \mathrm{C}$ entre cada ciclo.

La reducción total de espesor fue de 2,1:1 lo que corresponde a una deformación equivalente de $\varepsilon \sim 0,85$, según el criterio de von Mises. El espesor final del material laminado fue $11,5 \mathrm{~mm}$ y el de las diferentes capas fue de $\sim 990 \mu \mathrm{m}$ para las de Al 7075 y $\sim 270 \mu \mathrm{m}$ para las de Al 1050. El material multicapa resultante ha sido referenciado en este estudio como ADH19.

Debido a las altas temperaturas empleadas durante el procesado y el lento enfriamiento del material multicapa hasta temperatura ambiente fue necesario realizar un tratamiento térmico para recuperar la excelente resistencia mecánica de la aleación Al 7075

Tabla I. Propiedades mecánicas a temperatura ambiente de las aleaciones de aluminio de partida

Table I. Mechanical properties at room temperature of the as-received aluminium alloys

\begin{tabular}{lcccc}
\hline Aleación & $\boldsymbol{\sigma}_{\max }(\mathbf{M P a})^{*}$ & $\boldsymbol{\sigma}_{\mathbf{0 . 2}}(\mathbf{M P a})^{*}$ & HV & Alargamiento (\%)* $^{*}$ \\
\hline Al 7075-T6 (D) & 545 & 475 & 188 & 8 \\
Al 1050-H24 (H) & 105 & 75 & 44 & 10 \\
\hline
\end{tabular}

* Información suministrada por el productor del aluminio a partir de ensayos de tracción. $\sigma_{\max }:$ Resistencia máxima; $\sigma_{0.2}$ : límite elástico; HV: microdureza Vickers; T6: tratamiento de solubilización seguido de temple y envejecimiento artificial hasta máxima dureza; H24: endurecimiento por deformación seguido de un recocido parcial $\left(240^{\circ} \mathrm{C}\right)$. 


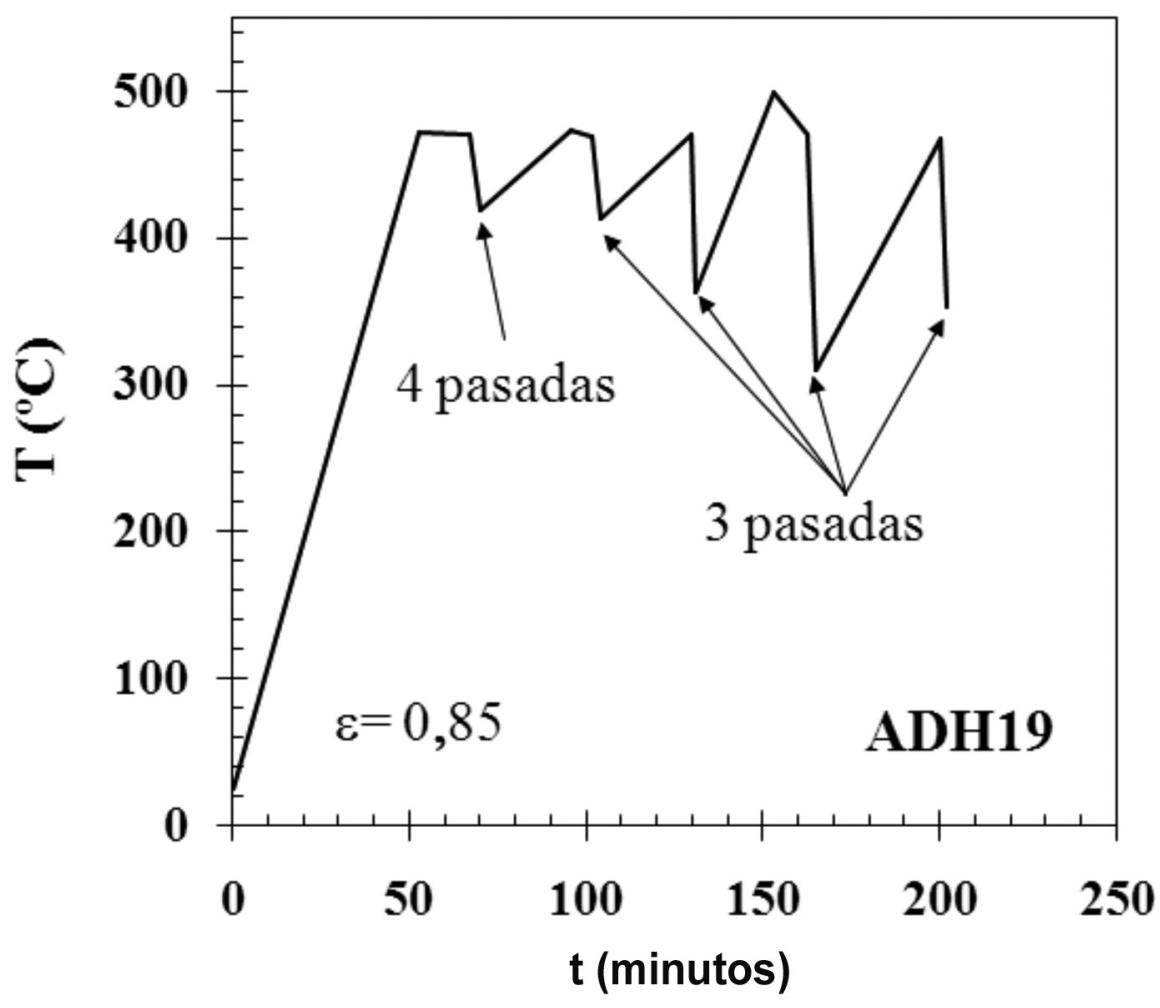

Figura 1. Esquema de la temperatura registrada durante el procesado del material laminado multicapa ADH19.

Figure 1. Scheme of the registered processing temperature of the ADH19 multilayer laminate.

presente en el laminado. El tratamiento térmico realizado fue el T6 que consiste en solubilización a $465{ }^{\circ} \mathrm{C}$ durante $30 \mathrm{~min}$, seguido de temple en agua fría y finalmente envejecimiento artificial durante $14 \mathrm{~h}$ a $135^{\circ} \mathrm{C}$.

\subsection{Caracterización microestructural}

En la figura 2 se muestra un esquema del material multicapa procesado, en el que se indican las direcciones y planos considerados tanto para la caracterización microestructural, como para el mecanizado de probetas para los correspondientes ensayos mecánicos.

La microestructura de las aleaciones de partida y contenidas en el material multicapa se analizó en la sección LT (longitudinal-transversal), la cual contiene la dirección de laminación y la normal al plano de laminación, prestando especial atención a la microestructura cerca de las intercaras. Para ello se realizaron micrografías de electrones retrodispersados en un microscopio electrónico de barrido (SEM) JEOL JSM 6500F equipado con filamento de emisión de campo.
La preparación metalográfica final tras las etapas convencionales de desbaste y pulido consistió en electropulido a $-15^{\circ} \mathrm{C}$ y $15 \mathrm{~V}$, utilizando como electrolito una disolución del $30 \%$ vol de $\mathrm{HNO}_{3}$ en $\mathrm{CH}_{3} \mathrm{OH}$.

La composición química cerca de las diferentes intercaras se determinó mediante espectroscopía de energía dispersiva de rayos-X (EDX) operando a $15 \mathrm{kV}$, y mediante el programa Oxford Inca.

\subsection{Ensayo de microdureza Vickers}

Se realizaron medidas de microdureza cerca de las diferentes intercaras en la sección LT, empleando un indentador Vickers (Matsuzawa Seiki MHT-1) con cargas de $100 \mathrm{~g}$ durante $15 \mathrm{~s}$. Los valores de microdureza Vickers se representaron frente a la distancia a la intercara para analizar los gradientes de propiedades mecánicas a través de diferentes intercaras. Tanto el tamaño de las huellas como la distancia desde su centro a la intercara se midieron mediante el programa de análisis de imagen SIGMA SCAN PRO. 


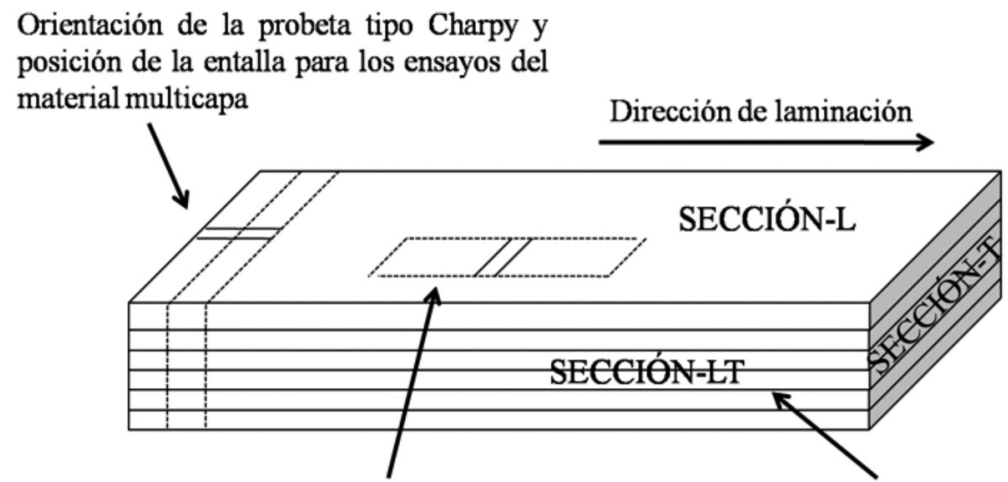

Orientación de la probeta tipo Charpy y posición de la entalla para los ensayos de los materiales de partida monolíticos
Plano (LT) de observación de la microestructura

Figura 2. Esquema que muestra los planos y orientaciones importantes para el estudio de la microestructura y para el mecanizado de la entalla en las probetas tipo Charpy para los ensayos de flexión en tres puntos e impacto.

Figure 2. Scheme showing planes and orientations that are important for studying the microstructures and for machining the notches in Charpy samples for three-point bend and impact tests.

\subsection{Ensayo de flexión en tres puntos}

Para caracterizar la respuesta mecánica de las intercaras y de las distintas capas, así como para realizar un seguimiento de la propagación de la grieta a través del material, se realizaron ensayos de flexión en tres puntos con probetas tipo Charpy $\left(10 \times 10 \times 55 \mathrm{~mm}^{3}\right)$, con entalla en $\mathrm{V}$ de $2 \mathrm{~mm}$ de profundidad. En la figura 2 se incluyen las orientaciones consideradas para el mecanizado de las probetas tipo Charpy y sus entallas tanto para el material multicapa, como para las aleaciones de partida monolíticas. Para el mejor aprovechamiento del laminado multicapa, las probetas se mecanizaron con la dirección más larga $(55 \mathrm{~mm})$ perpendicular a la dirección de laminación (Fig. 2). Posteriormente, para poder ensayar las probetas en la orientación en serie, que es aquélla en la que la grieta se propaga secuencialmente a través de cada una de las capas del laminado, y es la disposición lógica para este tipo de materiales en una posible aplicación en la industria aeroespacial, la entalla se mecanizó paralela a la dirección de laminación. En el caso de las aleaciones de partida monolíticas, donde no hay intercaras, pero sí contienen una microestructura de granos alargados en la dirección de laminación que puede contribuir a su tenacidad intrínseca al ser ensayadas en la orientación más favorable, la entalla se mecanizó paralela al plano y perpendicular a la dirección de laminación (Fig. 2).
El ensayo se realizó en una máquina universal de ensayos Servosis, a una velocidad del travesaño de $0,04 \mathrm{~mm} / \mathrm{s}$. Los resultados obtenidos a partir de este ensayo se representan mediante curvas carga-desplazamiento. Para comparar con la energía absorbida en el ensayo de impacto Charpy se calculó la energía absorbida en el ensayo de flexión como el área bajo la curva F-d hasta un desplazamiento del punzón de $20 \mathrm{~mm}$, que corresponde al valor de deformación necesaria para que la probeta forme un ángulo de $70^{\circ}$, aproximadamente igual al que se obtiene en un ensayo de impacto Charpy.

\subsection{Ensayo de impacto Charpy}

Para estudiar la tenacidad a impacto tanto de las aleaciones de partida como del material multicapa procesado, se realizaron ensayos de impacto con un péndulo Charpy con una capacidad máxima de $294 \mathrm{~J}$. Las muestras consideradas fueron las descritas anteriormente en el ensayo de flexión, y los ensayos se realizaron también en la orientación en serie. Se ensayaron tres muestras de cada uno de los materiales estudiados.

Finalmente, las muestras fracturadas bajo diferentes condiciones de ensayo (flexión y Charpy) se examinaron mediante microscopía óptica y electrónica de barrido, para evaluar los mecanismos de fractura responsables del aumento de tenacidad. 


\section{RESULTADOS Y DISCUSIÓN}

\subsection{Microestructura}

La figura 3 muestra la microestructura de las aleaciones de partida en la sección LT. La figura 3 a) corresponde a la aleación Al 7075-T6 donde se pueden observar granos alargados en la dirección de laminación (20-30 $\mu \mathrm{m})$, con un espesor en la dirección normal de $\sim 10 \mu \mathrm{m}$.

Por otra parte, la figura $3 \mathrm{~b}$ ) muestra la microestructura correspondiente al aluminio de pureza comercial Al 1050-H24, donde se observa una microestructura constituida por (sub)granos equiaxiales con un tamaño medio de 2-3 $\mu \mathrm{m}$. Adicionalmente, en ambas muestras se observan partículas de compuestos intermetálicos ricas en hierro distribuidas al azar. Estos compuestos intermetálicos oscilan en tamaño entre 0,5-5 $\mu \mathrm{m}$. En el caso del Al 7075, los precipitados de $\mathrm{MgZn}_{2}$, los cuales son los principales responsables de su alta resistencia mecánica ${ }^{[16]}$, no pueden ser observados mediante SEM debido a su tamaño nanométrico.

La microestructura del material multicapa laminado se muestra en la figura 4 .

La figura 4 a) muestra una micrografía general del laminado ADH19, donde se observa que está constituido por capas con una microestructura de granos muy grandes $(\sim 50-60 \mu \mathrm{m})$ correspondientes a las capas de $\mathrm{Al} 1050$ (H) (Fig. 4 c) a mayores aumentos), alternadas con capas de Al 7075 (D) constituidas por granos finos y alargados en la dirección de laminación, formando una microestructura tipo oblea o "pancake". La figura $4 \mathrm{~d}$ ) muestra una micrografía a mayores aumentos de la aleación Al 7075 (D), donde se observa con mayor detalle que el espesor de los granos iniciales se ha reducido hasta $\sim 3-4 \mu \mathrm{m}$ en el laminado como consecuencia de la deformación plástica. Además, estos granos alargados han sido subdivididos, formando una (sub)estructura prácticamente equiaxial con un tamaño medio de $\sim 2-3 \mu \mathrm{m}$. Por tanto, la microestructura de la aleación Al 7075 (D) en el laminado multicapa es considerablemente más fina que en el material de partida (Fig.3 a)). Sin embargo, en el caso del Al 1050 (H) (Fig.4 c)) se ha producido un crecimiento considerable del grano. La presencia de elementos aleantes como cinc, magnesio y cobre en el Al 7075, junto con los correspondientes precipitados que se forman inducidos por la deformación y durante el enfriamiento posterior, fijan las fronteras de grano, estabilizando la microestructura y evitando una posible recristalización durante el procesado o el posterior tratamiento T6. Por el contrario, el Al 1050 sólo contiene pequeñas cantidades de hierro y silicio, los cuales forman compuestos intermetálicos insolubles a las temperaturas consideradas en el presente estudio que no pueden evitar la recristalización discontinua que tiene lugar durante el procesado en esta aleación por encima de $400^{\circ} \mathrm{C}$ dada su pequeña fracción de volumen.

Los cambios microestructurales observados en ambas aleaciones como consecuencia del procesado contribuirán a mejorar la tenacidad intrínseca del laminado. Por una parte, la microestructura más fina del Al 7075 (D) favorecerá una mayor resistencia mecánica de acuerdo con la conocida relación de Hall-Petch, mientras que el gran tamaño de grano libre de la acritud de partida del Al 1050 (H) contribuirá a aumentar la ductilidad de estas capas, dificultando la propagación y renucleación de la grieta a través de ellas.

Por otro lado, la figura 4 b) muestra una micrografía SEM de la intercara formada entre dos capas
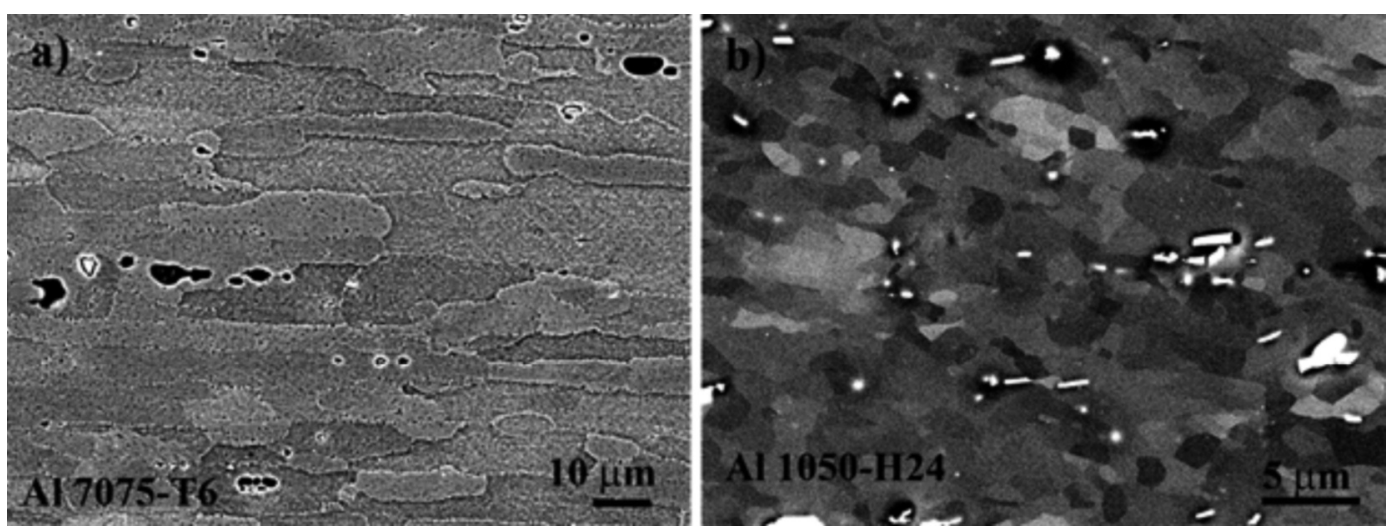

Figura 3. Micrografías SEM de la sección longitudinal-transversal (LT) de las aleaciones de partida: a) Al 7075-T6; b) Al 1050-H24.

Figure 3. SEM micrographs of the longitudinal-transversal (LT) section of the as-received alloys: a) Al 7075-T6; b) Al 1050-H24. 

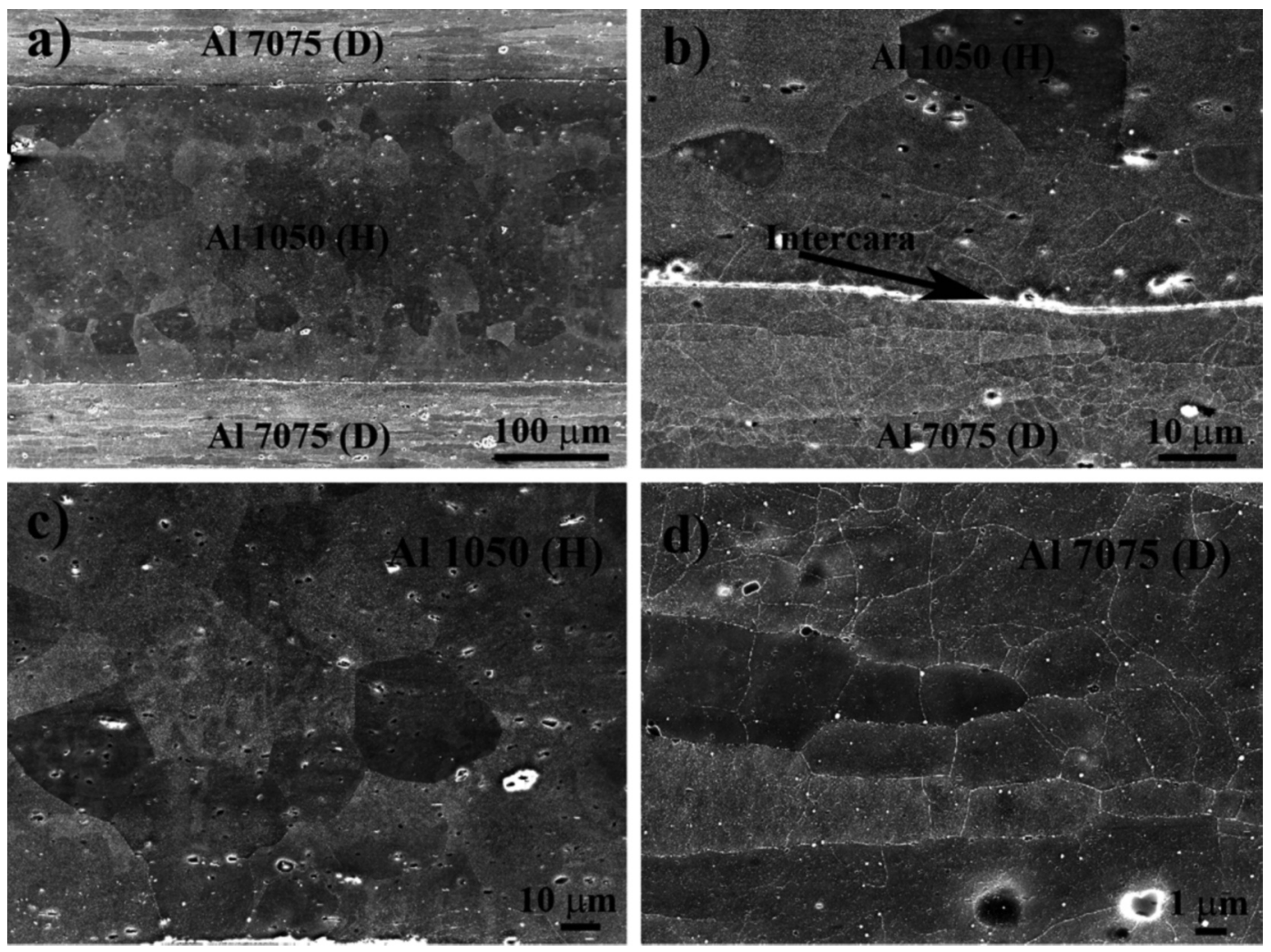

Figura 4. Micrografías SEM de secciones LT del laminado multicapa ADH19 tomadas a diferentes aumentos: a) Vista general del laminado donde se observa una capa de Al 1050 situada entre dos capas de AI 7075; b) Intercara entre dos capas de aluminio; c) Capa de Al 1050; d) Capa de Al 7075.

Figure 4. SEM micrographs of $L T$ sections of the $A D H 19$ multilayer laminate at different magnifications: a) General view showing an Al 1050 layer between two Al 7075 layers; b) Interface between aluminium layers; c) Al 1050 layer; d) Al 7075 layer.

de aluminio. Se puede observar la presencia de partículas blancas y brillantes, que corresponden a partículas de alúmina, surgidas de la fractura de la capa natural de óxido presente en las aleaciones de partida. Durante el procesado de laminación, el aluminio es capaz de deformar plásticamente y extenderse en función del grado de reducción impuesto. Sin embargo, la capa de óxido de aluminio es frágil y la respuesta a la deformación es fracturarse en partículas alineadas en la dirección de laminación, cuyo tamaño se relaciona con la tensión aplicada y el grado de deformación ${ }^{[17]}$. El aluminio durante la deformación extruye entre las grietas abiertas en la capa de alúmina, dando lugar a la unión entre capas. Por tanto, la intercara es una combinación de fragmentos de alúmina y aluminio extruido, que son las áreas pegadas entre capas, y esta combinación jugará un papel muy importante en las propiedades mecánicas de las intercaras, pudiendo tener lugar mecanismos extrínsecos como la delaminación, que será el principal mecanismo de frenado de la grieta en estos materiales multicapa.
También se puede observar junto a la intercara (Fig. 4 b)), y especialmente en la capa de $\mathrm{Al} 1050$ (H), un gradiente de tamaño de grano, siendo más fino cerca de la intercara. Este gradiente es debido a los procesos de recristalización que tienen lugar en el material durante el procesado como consecuencia de la alta temperatura y la deformación, y está asistido por la difusión de elementos aleantes desde el Al 7075 hacia el Al 1050. Los precipitados formados como consecuencia de dicha difusión ${ }^{[16]}$ son más numerosos cerca de la intercara, fijando las fronteras de grano y dificultando su crecimiento.

\subsection{Gradientes de composición y ensayo de microdureza Vickers}

En la figura 5 se muestra el microanálisis realizado cerca de diferentes intercaras así como las medidas de microdureza Vickers realizadas a través de las mismas intercaras. 


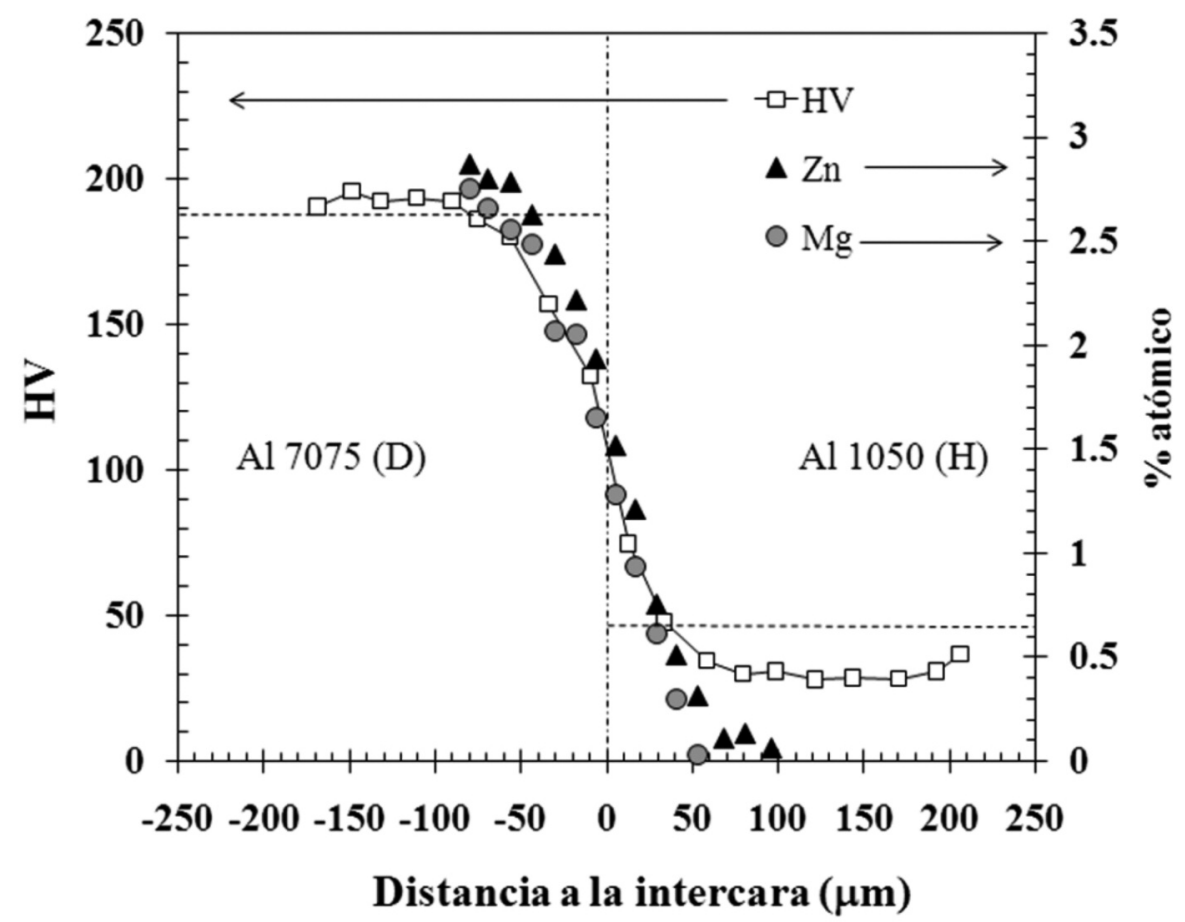

Figura 5. Microdureza Vickers (100 g/15 s) y porcentaje atómico de elementos aleantes ( $\mathrm{Zn}$ y $\mathrm{Mg}$ ) cerca de la intercara en el laminado multicapa ADH19.

\section{Figure 5. Vickers microhardness (100 g/15 s) and atomic percentage of alloying elements ( $\mathrm{Zn}$ y $\mathrm{Mg}$ ) close to the interface in the ADH19 multilayer laminate.}

En un artículo anterior ${ }^{[18]}$, donde se estudió la influencia del aluminio puro 1050 constreñido entre dos aleaciones de aluminio de alta resistencia, se observó que la extensión del gradiente de elementos aleantes determina las propiedades mecánicas de las aleaciones constituyentes cerca de la intercara. Como ya se ha mencionado, la presencia de precipitados $\mathrm{MgZn}_{2}$ es la principal responsable de las propiedades mecánicas de la aleación Al 7075. Como se puede observar en la figura 5, el gradiente de concentración de los principales aleantes (cinc y magnesio) en el Al 7075 se produce en una zona que se extiende aproximadamente 70-80 $\mu \mathrm{m}$ a ambos lados de la intercara, coincidiendo exactamente con la variación de la dureza a través de la misma. Además, se han realizado medidas tanto de composición como de microdureza a través de diferentes intercaras, más externas o internas, y no se han observado diferencias en función de su localización en el laminado. La figura muestra el refuerzo por endurecimiento por precipitación en la zona cercana a la intercara del Al $1050(\mathrm{H})$ que, como se ha comentado, favorece el afino de grano observado (Fig. 4 b)). Por el contrario, la pérdida de elementos aleantes capaces de formar precipitados endurecedores reduce sustancialmente la resistencia mecánica del Al 7075 cerca de la intercara, a pesar de haberse producido un notable afino de grano como consecuencia de la deformación.

Por otro lado, las líneas punteadas en la figura 5 corresponden a los valores de microdureza de las aleaciones en su estado inicial, siendo $188 \mathrm{HV}$ para el Al 7075-T6 y 44 HV para el Al 1050-H24. Después del procesado de laminación, la aleación Al 7075 muestra un valor de microdureza promedio en las zonas alejadas de la intercara de $192 \mathrm{HV}$, que es ligeramente superior al valor inicial. Este aumento de dureza se atribuye al afino de grano producido durante el procesado del material multicapa. Por el contrario, el Al $1050(\mathrm{H})$ muestra un valor de microdureza de $29 \mathrm{HV}$, el cual es considerablemente menor que el valor inicial (44 HV), y es debido al engrosamiento de su microestructura y la disminución de la densidad de dislocaciones ocasionada por la alta temperatura durante el procesado y el posterior tratamiento térmico T6. Hay que tener en cuenta que la aleación de partida Al 1050 en el estado H24 mantiene un cierto grado de acritud que incrementa su dureza respecto del Al 1050 recocido a mínima dureza. 


\subsection{Ensayo de flexión en tres puntos}

De forma tradicional, el ensayo de impacto Charpy se ha utilizado para evaluar la tenacidad relativa de los materiales ingenieriles y su sensibilidad a la entalla. En este estudio se ha considerado adicionalmente el uso del ensayo de flexión en tres puntos porque aporta mucha más información sobre el comportamiento de los materiales multicapa que el ensayo Charpy. Permite seguir de forma gráfica la propagación de la grieta a través del material multicapa procesado, determinando el frenado de las grietas en las intercaras, posibles delaminaciones o la renucleación de una nueva grieta. Además, permite comparar valores de tenacidad a través de la relación entre áreas bajo las curvas carga-desplazamiento. Posteriormente se compararán los valores de tenacidad para los materiales considerados en este trabajo obtenidos mediante ensayo de impacto Charpy y mediante el cálculo del área bajo la curva carga-desplazamiento obtenida mediante ensayos de flexión.

La figura 6 a) y b) muestra una macrografía del ensayo de flexión realizado, y de la disposición de la probeta del material multicapa sobre los apoyos de flexión.

Desde el momento en que se empieza a aplicar carga sobre el material laminado, todas las intercaras están sometidas a diferente grado de deformación, de tal forma que su respuesta a tal deformación será la principal responsable del incremento de tolerancia al daño del material multicapa. Con la finalidad de analizar la respuesta individual de las diferentes intercaras a la aplicación de tensión-deformación durante el ensayo de flexión, se realizó un ensayo interrumpido cuya curva carga-desplazamiento se muestra en la figura $6 \mathrm{c}$ ). La figura 6 b) muestra un detalle de la muestra ensayada, donde se observa que después de la fractura de la capa en la que se encuentra la punta de la entalla se produce el daño en una intercara interior. La curva carga-desplazamiento (Fig. 6 c)), obtenida hasta el momento en el que se produce este daño en una intercara interior, muestra una leve caída de carga que genera una discontinuidad en el laminado, la cual hemos definido como una pre-delaminación en dicha intercara, y como se discutirá a continuación, será muy beneficiosa para el aumento de la tolerancia al daño del laminado.

La figura 7 muestra las curvas carga-desplazamiento correspondientes a las aleaciones de partida monolíticas (Al-7075-T6 y Al-1050) y tres muestras del laminado ADH19, dos de ellas ensayadas hasta la rotura total del laminado y otra sometida al ensayo de flexión interumpido comentado anteriormente. El Al 7075-T6 de partida muestra alto valor de carga $(10 \mathrm{kN})$, pero baja ductilidad. Por el contrario, el Al 1050 muestra baja carga $(2,5 \mathrm{kN})$ pero excelente ductilidad.

Por otro lado, el material multicapa ADH19 presenta una carga máxima a la flexión de $7,9 \mathrm{kN}$, siendo similar a la que le correspondería de acuerdo a la regla de la mezclas $(8,0 \mathrm{kN})$. Esto es debido a que el aumento de resistencia mecánica que experimenta
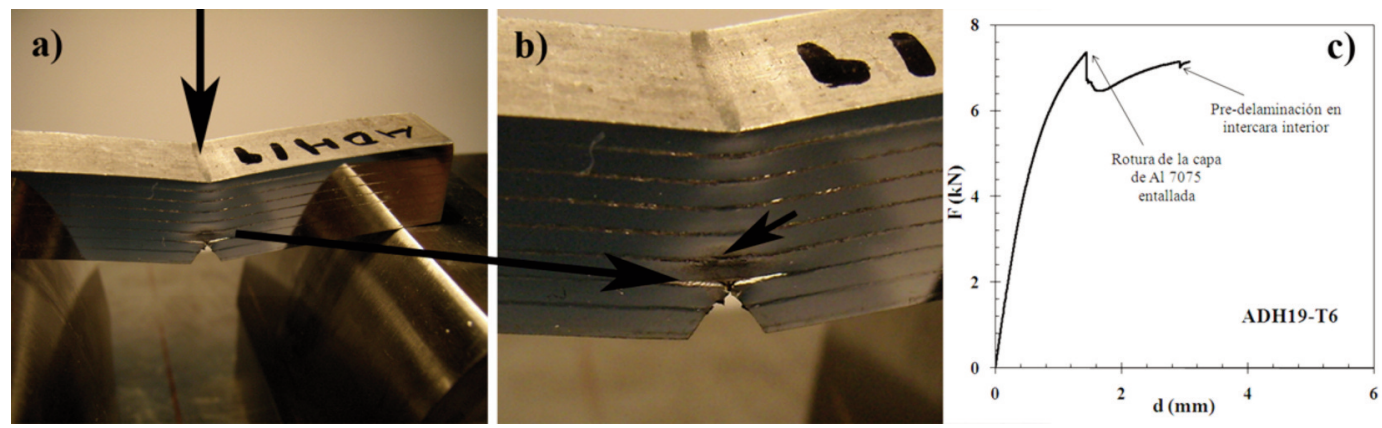

Figura 6. a) Macrografía de una muestra del laminado ADH19 ensayada mediante flexión en tres puntos; b) Detalle a mayores aumentos de la muestra ensayada a flexión, mostrando el daño y la deformación que experimenta una intercara interior más allá del frente de la grieta; c) Curva carga-deformación obtenida mediante el ensayo de flexión, el cual se detuvo cuando se detectó una caída de carga correspondiente a una pre-delaminación en una intercara interior.

Figure 6. a) Macrograph of an ADH19 sample tested by three-point bending; b) Detail at higher magnification of the bend tested sample showing the damage and deformation experimented by an inner interface beyond the crack front; c) Loaddisplacement curve obtained by the bend test, which was stopped when a load step corresponding to the pre-delamination of an inner interface was observed. 


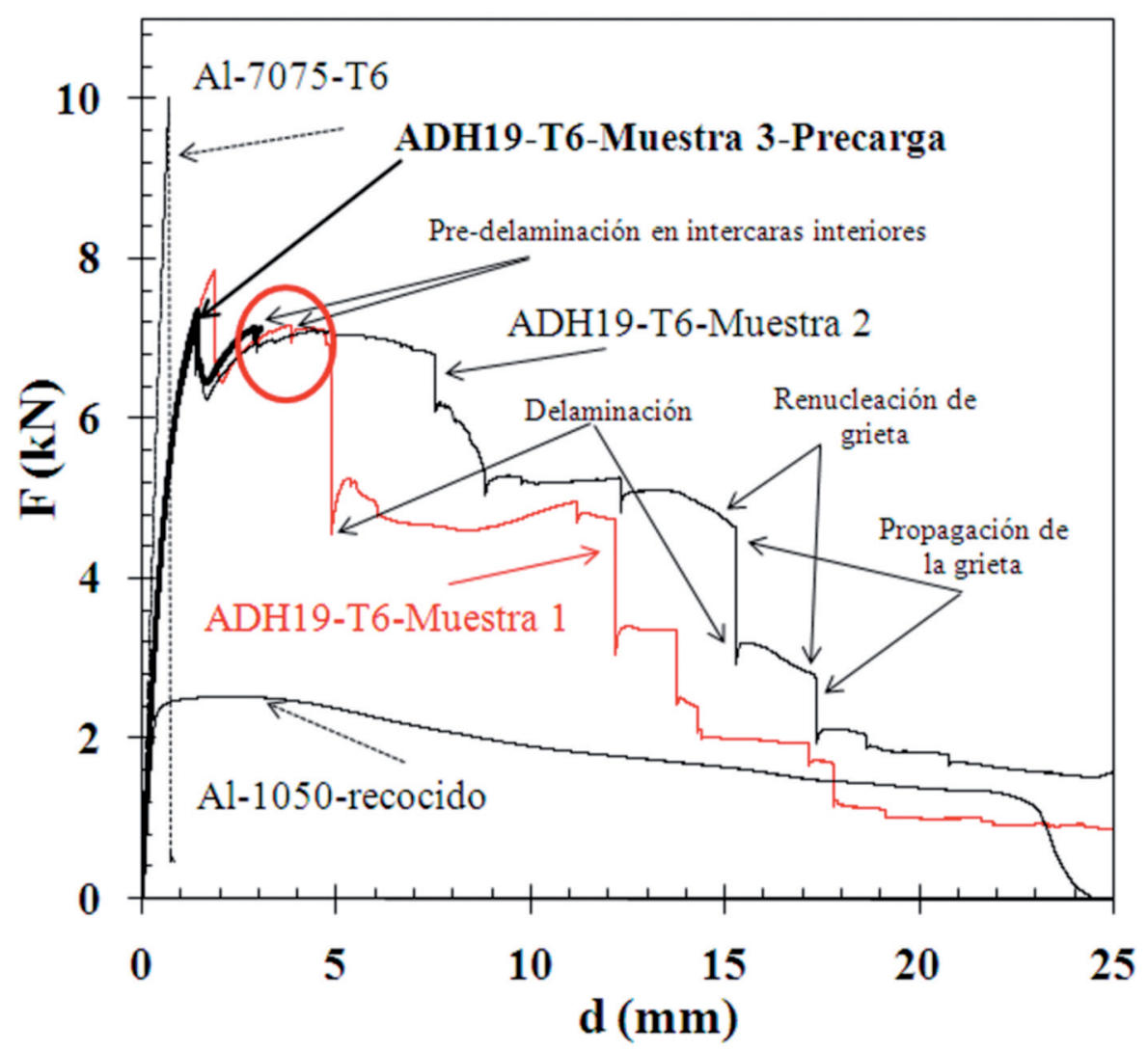

Figura 7. Curvas carga-desplazamiento obtenidas a partir de ensayos de flexión en tres puntos de la aleación de partida Al 7075-T6, Al 1050 recocido y diferentes muestras del laminado multicapa ADH19 en la orientación en serie.

Figure 7. Load-displacement curves obtained from three-point bend tests of notched as-received Al 7075-T6, annealed Al 1050 and different samples of the ADH19 multilayer laminate in crack-arrester orientation.

el Al 7075 por afino de grano durante el procesado, medido mediante microdureza Vickers (Fig. 5), compensa el ablandamiento que experimenta la aleación Al 1050 por la pérdida de la acritud inicial y engrosamiento del tamaño de grano, y que también se ha observado a raíz del ensayo de dureza.

Por otra parte, el material multicapa presenta una excelente ductilidad, siendo incluso superior a la del $\mathrm{Al}$ 1050. Las tres muestras del laminado multicapa ensayadas presentan similar resistencia máxima y las dos muestras ensayadas hasta rotura presentan similar ductilidad, indicando gran reproducibilidad en los resultados obtenidos.

El aumento de ductilidad es debido a los mecanismos de fractura, tanto intrínsecos como extrínsecos, que operan durante el ensayo de flexión. Las tres curvas del material multicapa presentan en primer lugar un endurecimiento por deformación hasta la primera caída de carga correspondiente a fractura de la capa en la que se sitúa la punta de la entalla. La caída brusca de carga nos indica que la entalla se encuentra en una capa de Al 7075, que debido a su fragilidad, una vez nucleada la grieta se propaga rápidamente hasta ser frenada en la siguiente capa de Al 1050 mucho más dúctil. Las capas de $\mathrm{Al} 1050$ frenan y ralentizan la propagación de la grieta mediante un mecanismo de tenacidad intrínseco, debido a la alta ductilidad del aluminio puro, disminuyendo la concentración de tensiones.

Por otra parte, la forma escalonada de las curvas F-d en la figura 7 revela que están operando mecanismos de fractura extrínsecos, como la delaminación en las intercaras, las cuales son responsables del extraordinario aumento de la tenacidad (área bajo la curva), porque paralelamente tras la delaminación tendrán lugar otros mecanismos que requieren gran consumo de energía como por ejemplo la renucleación de nuevas grietas.

Las delaminaciones provocan que la renucleación de la siguiente grieta principal se produzca en una 
zona mucho más deslocalizada, de mayor volumen, necesitando un extra de endurecimiento por deformación, y una gran deformación plástica para seguir fracturando y avanzando la grieta. En consecuencia, las delaminaciones son claramente identificadas en las curvas F-d porque tras las caídas de carga correspondientes a la fractura de una capa se produce un pequeño endurecimiento por deformación del material restante, y un rellano en la curva hasta que se alcanza una deformación crítica para la renucleación de una nueva grieta que dará lugar a una nueva caída de carga. El resultado es un notable ensanchamiento de la curva de flexión, con los extensos rellanos observados, y por consiguiente una gran tenacidad total.

Adicionalmente, las tres muestras ensayadas del material multicapa presentan pequeñas caídas de carga las cuales, como se ha comentado, se han analizado mediante la realización de un ensayo de flexión interrumpido y se ha determinado que corresponden a pequeñas delaminaciones en intercaras interiores producidas antes de que la grieta principal las alcance (Fig. 6 b)). Este mecanismo de fractura que hemos definido como pre-delaminación interfacial ha sido visto previamente en otras configuraciones de materiales multicapa ${ }^{[7]}$.

En la figura 8, mediante una composición de micrografías ópticas, se muestra la secuencia mencionada de propagación de la grieta a través del laminado, desde su nucleación en la punta de la entalla (1) hasta ser enromada y frenada en la capa de Al 1050 (3). También se observa la presencia de una pequeña pre-delaminación (4) en una intercara interior, a cierta distancia de la grieta principal.

Estas pre-delaminaciones se producen debido a la fragilidad de las intercaras por la presencia de las partículas de alúmina, y a la redistribución de tensiones que se produce durante la deformación. Cabe mencionar que en los materiales multicapa, debido a la propagación de grietas a lo largo de intercaras, así como por la diferente ductilidad de las aleaciones constituyentes del laminado, se genera una desloca-

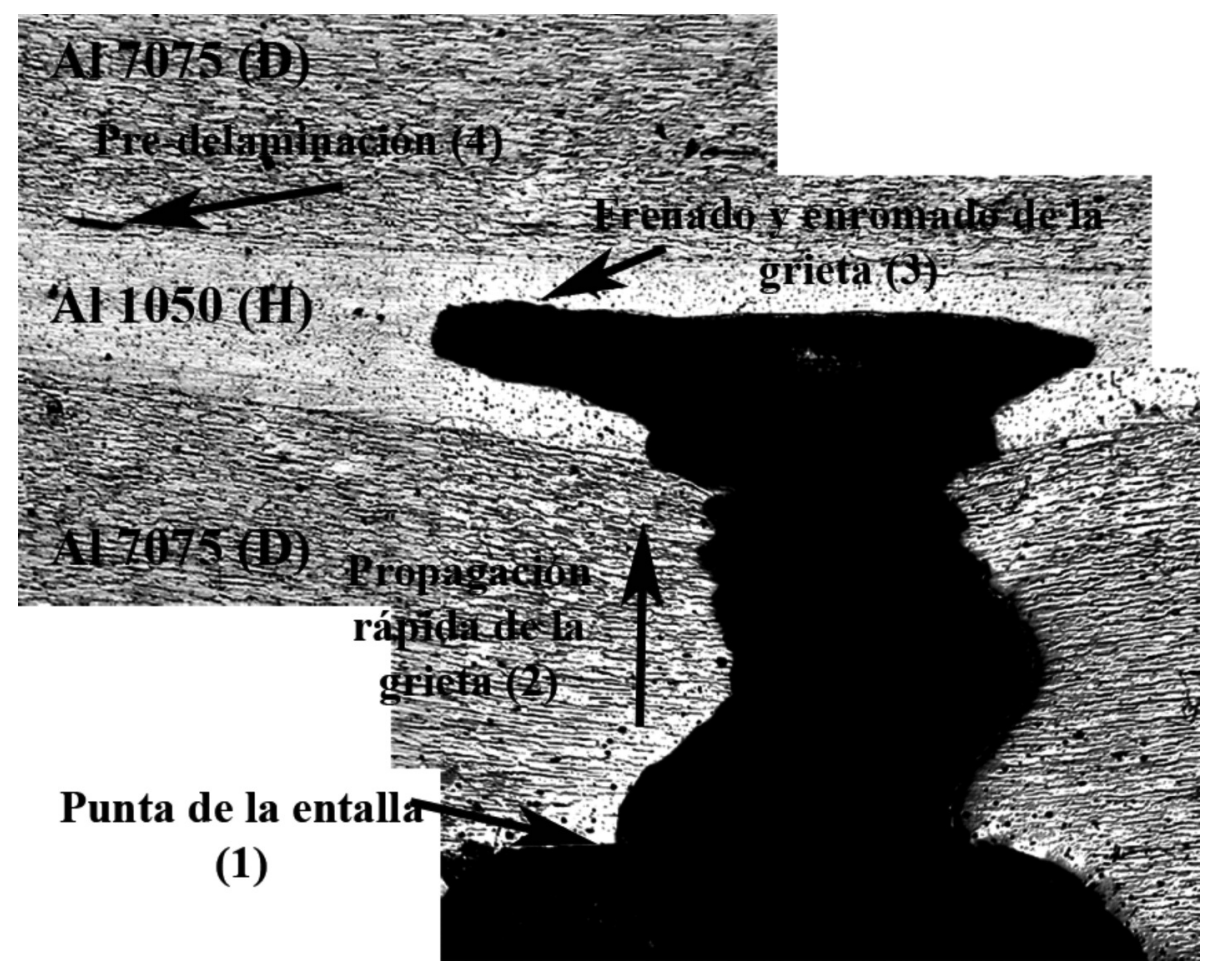

Figura 8. Composición de micrografías ópticas de una muestra del laminado ADH19 ensayada mediante flexión, donde se muestra la secuencia de propagación de la grieta a través de los materiales constituyentes desde su nucleación en la punta de la entalla (1), hasta la pre-delaminación (4) de una intercara interior.

Figure 8. Optical micrographs composition of a bend tested ADH19 laminate sample showing the crack propagation sequence across the constituent materials from the crack nucleation (1) at the notch tip until pre-delamination (4) in an inner interface. 
lización de la tensión, no estando concentrada en el plano que contiene la dirección de la carga durante el ensayo.

Así, la pre-delaminación garantiza una mayor delaminación y renucleación de nuevas grietas conforme la deformación continúa y como resultado pueden ocurrir otros mecanismos extrínsecos como es el puenteo de la grieta ("crack bridging"[19]). Este mecanismo tiene lugar cuando se generan nuevas grietas en puntos relativamente alejados de la grieta principal, permaneciendo entre ambos puntos un ligamento dúctil que dificulta el crecimiento de dicha grieta principal. De tal forma en el presente laminado, la capa situada entre la primera delaminación y la siguiente intercara pre-delaminada actúa como un ligamento sin fracturar, el cual si además es de $\mathrm{Al} 1050$ con alta ductilidad, requiere una gran deformación plástica para renuclear una nueva grieta en dicha capa, con la adicional absorción de energía que eso conlleva. Sin embargo, mientras las capas de Al 1050 deforman y endurecen, en las capas de Al 7075 mucho más frágiles se generan nuevas grietas por su menor resistencia a la flexión. Además, conforme la deformación continúa, la longitud delaminada en las intercaras se incrementa, lo cual contribuye aún más a deslocalizar la tensión aplicada, de forma que ésta no se encuentra localizada en la línea de avance de la grieta principal, siendo necesaria más deformación y tensión para fracturar las distintas capas. Esto produce un adicional aumento de la resistencia del material debido al endurecimiento por deformación. En definitiva, la secuencia simultánea de los mecanismos de fractura descritos justifica las diferentes caídas de carga que se observan en las curvas de flexión.

Por tanto, si el objetivo es la tenacidad del material multicapa, no son deseables intercaras excesivamente unidas, es decir, muy tenaces, y se prefieren delaminaciones controladas.

\subsection{Ensayo de impacto Charpy}

Finalmente, los resultados del ensayo de impacto Charpy se incluyen en la tabla II.

También se incluyen en la tabla II los valores de tenacidad calculados como el área bajo las curvas F-d del ensayo de flexión, para comparación con los resultados obtenidos en el ensayo Charpy. El valor medio de energía absorbida en el ensayo Charpy para el Al 7075-T6 (D) de partida monolítico es $62 \mathrm{~kJ} / \mathrm{m}^{2}$, mientras que para el Al 1050-H24 (H) es $333 \mathrm{~kJ} / \mathrm{m}^{2}$. El material multicapa ADH19-T6 muestra una tenacidad a impacto considerablemente mayor, presentando un valor medio de $1.095 \mathrm{~kJ} / \mathrm{m}^{2}$, el cual es 17,7 y 3,3 veces superior a los valores correspondientes a las aleaciones de partida Al 7075-T6 y Al 1050-H24, respectivamente. Por otra parte, los valores obtenidos mediante el ensayo de flexión son $55 \mathrm{~kJ} / \mathrm{m}^{2}$ para el Al 7075-T6 (D), $230 \mathrm{~kJ} / \mathrm{m}^{2}$ para el Al $1050 \mathrm{y}$ $1.167 \mathrm{~kJ} / \mathrm{m}^{2}$ para el material multicapa ADH19-T6. Por tanto, los valores de tenacidad obtenidos mediante ambos métodos son del mismo orden de magnitud y presentan la misma tendencia para los tres materiales considerados. Cabe destacar que, sin embargo, entre ambos métodos existen diferencias que justifican las discrepancias encontradas en los valores de tenacidad. Así, la diferencia fundamental entre ambos métodos de ensayo es la velocidad de deformación, la cual es $\sim 1.2 \times 10^{-3} \mathrm{~s}^{-1}$ para el ensayo de flexión, y considerablemente más alta, $\sim 1.5 \times 10^{2} \mathrm{~s}^{-1}$, para el ensayo de impacto Charpy.

Por otra parte, el espectacular aumento de la tenacidad en el material multicapa procesado es debido a que la configuración de materiales diseñada, junto con el procesado adecuado, ha conducido a un material de elevada resistencia y excelente ductilidad. La combinación de mecanismos intrínsecos (alta ductilidad del Al 1050 y mayor resistencia del Al 7075), y extrínsecos (delaminaciones, puenteo

Tabla II. Energía absorbida durante el ensayo de impacto Charpy y el ensayo de flexión en tres puntos de las aleaciones de partida y del material multicapa ADH19-T6

Table II. Absorbed energy of V-notched samples of the as-received alloys and the ADH19-T6 multilayer laminate from Charpy and three-point bend tests

\begin{tabular}{lrrr}
\hline \multicolumn{1}{c}{ Material } & $\begin{array}{c}\text { Resiliencia }\left(\mathbf{k J} / \mathbf{m}^{2} \text { ) }\right. \\
\text { (Charpy) }\end{array}$ & $\begin{array}{c}\text { Resiliencia (kJ/m²) } \\
\text { (Flexión) }\end{array}$ \\
\hline Al 7075-T6 (D) & $62 \pm 2$ & $55 \pm 3$ \\
Al 1050-H24 (H) & $333 \pm 18$ & $230 \pm 12$ \\
ADH19-T6 & $1.095 \pm 65$ & $1.167 \pm 188$ \\
\hline
\end{tabular}



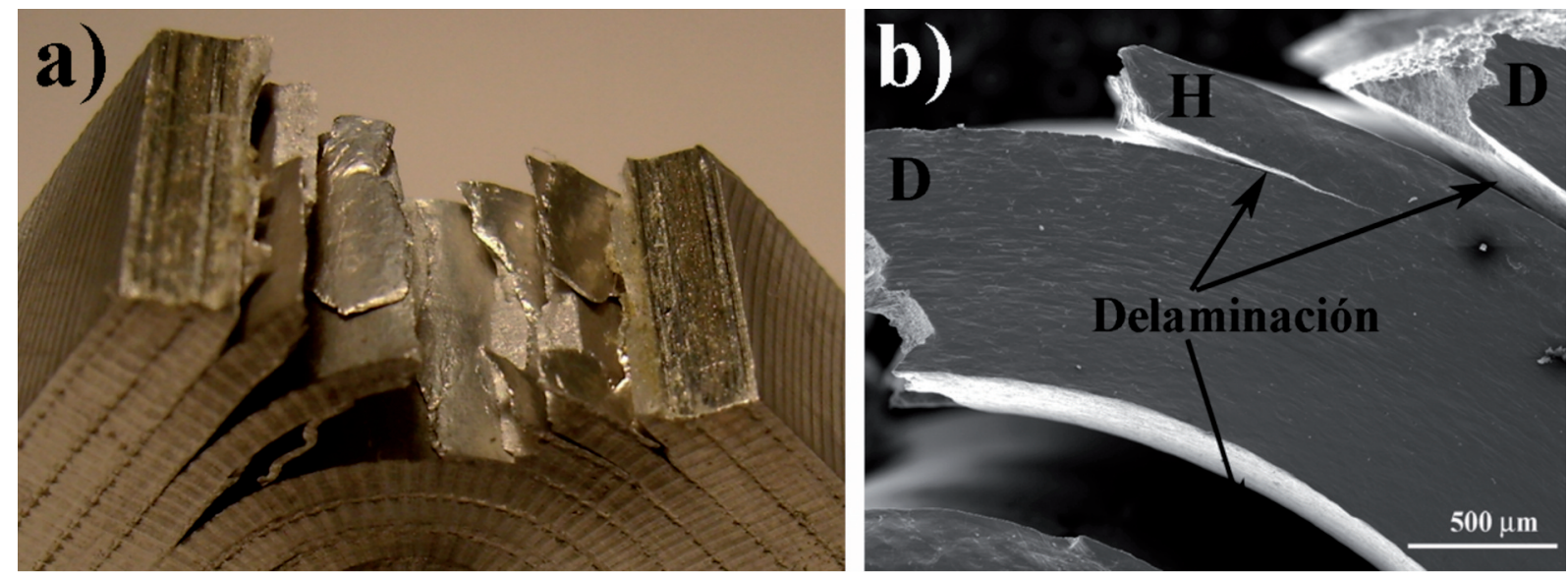

Figura 9. a) Macrografía y b) micrografía SEM de una muestra fracturada del laminado ADH19 después de un ensayo de impacto Charpy.

\section{Figure 9. a) Macrograph and b) SEM micrograph of a fractured sample of the ADH19 laminate after Charpy impact test.}

de grieta y capas/ligamentos sin fracturar) previenen la propagación catastrófica de la grieta, y son los responsables de la alta tolerancia al daño del material multicapa ADH19, como se ha visto en los ensayos de flexión.

La figura 9 a) corresponde a una macrografía de una muestra ensayada mediante impacto Charpy, donde se muestra el estado final del laminado al ser sometido a deformación a alta velocidad.

A mayores aumentos, mediante una micrografía SEM (Fig. 9 b)) se observa cómo todas las intercaras han delaminado y la extensiva deformación plástica que han experimentado las capas de Al 1050 y Al 7075 para renuclear nuevas grietas. De acuerdo a las curvas F-d mostradas en la figura 7 para el material multicapa vemos que la magnitud de la carga que soporta el material en cada momento depende del espesor del laminado que queda sin fracturar después de la delaminación. Sin embargo, la longitud de la delaminación es un factor importante que determina el incremento de carga que se va a producir por endurecimiento debido a la deformación del resto de material sin fracturar, así como la deformación plástica necesaria para renuclear una nueva grieta. Por tanto, cuanto mayor sea la delaminación mayor será la tolerancia al daño y la energía absorbida durante la fractura.

Finalmente, a partir de los resultados presentados se demuestra que en el material multicapa se produce una compleja combinación de mecanismos extrínsecos e intrínsecos resultado de la configuración de las aleaciones seleccionadas y el procesado realizado. Sin embargo, el mecanismo extrínseco debido a las intercaras propensas a delaminar es el principal responsable del fuerte aumento de la tenacidad.

\section{CONCLUSIONES}

- En el presente trabajo se ha procesado un material compuesto de 19 capas alternadas de las aleaciones $\mathrm{Al} 7075$ (82\% vol) y Al 1050 (18\% vol) mediante laminación en caliente. El material multicapa resultante presenta excelente combinación de resistencia y ductilidad, lo que supone una elevada tolerancia al daño, la cual ha sido 18 veces superior a la de la aleación de aluminio aeroespacial Al 7075 de partida. Esta extraordinaria tenacidad a impacto es fruto de la configuración de las aleaciones seleccionadas que junto con el procesado han potenciado mecanismos de tenacidad tanto intrínsecos como extrínsecos.

- La mejora de la tenacidad por mecanismos intrínsecos se debe al aumento de la resistencia del Al 7075 con un tamaño de grano más fino, y a la gran ductilidad del Al 1050. Sin embargo, son los mecanismos extrínsecos los que contribuyen drásticamente al aumento de la tenacidad, destacando la delaminación en las intercaras, pequeñas pre-delaminaciones en intercaras interiores, renucleación y puenteo de grietas.

\section{Agradecimientos}

Los autores agradecen al MICINN la financiación recibida a través del proyecto MAT2009-14452. 


\section{REFERENCIAS}

[1] S. Kikuchi, H. Kuwahara, N. Mazaki, S. Urai y H. Miyamura, Mater. Sci. Eng. A 234-236 (1997) 1.114-1.117.

[2] A.B. Pandey, B.S. Majumdar y D.B. Miracle, Acta Mater. 49 (2001) 405-417.

[3] V. Braccini, D. Marré, A. Malagoli, A. Mollica, M. Putti, G. Balestrino, S. Lavanga, P.G. Medaglia y A.S. Siri, Physica C, 372-376 (2002) 616-618.

[4] J. Torrejón, G. Badini, K. Pirota y M. Vázquez, Acta Mater. 55 (2007) 4.271-4.276.

[5] A. Pegoretti, I. Cristelli y C. Migliaresi, Compos. Sci. Technol. 68 (2008) 2.653-2.662.

[6] F. Roudolff y M. Gädke, Aerosp. Sci. Technol. 4 (2000) 23-32.

[7] C.M. Cepeda-Jiménez, M. Pozuelo, J.M. GarcíaInfanta, O.A. Ruano y F. Carreño, Metall. Mater. Trans. A 40 (2009) 69-79.

[8] J.D. Embury, N.J. Petch, A.E. Wraith y E.S. Wright, Trans. Metall. Soc. AIME, 239 (1967) 114-118.

[9] D.W. Kum, T. Oyama, J. Wadsworth y O.D. Sherby, J. Mech. Phys. 31 (1983) 173-186.
[10] D.W. Kum, T. Oyama, O.A. Ruano y O.D. Sherby, Metall. Trans. A, 17 (1986) 1.517-1.521.

[11] J. Wittenauer, O.D. Sherby, J. Eng. Mater. Technol., Trans. ASME, 109 (1987) 244-251.

[12] F. Carreño, M. Pozuelo, J. Chao, O.A. Ruano, Rev. Metal. Madrid 37 (2001) 130-134.

[13] F. Carreño, J. Chao, M. Pozuelo, O.A. Ruano, Scripta Mater. 48 (2003) 1.135-1.140.

[14] J.E. Hatch (Ed.), Aluminum. Properties and Physical Metallurgy, American Society for Metals, Metals Park, OH, 1984, pp.370.

[15] C.M. Cepeda-Jiménez, M. Pozuelo, J.M. GarcíaInfanta, O.A. Ruano y F. Carreño, Mater. Sci. Eng. A 496 (2008) 133-142.

[16] F. Viana, A.M.P. Pinto, H.M.C. Santos y A.B. Lopes, J. Mater. Process. Technol., 92-93 (1999) 54-59.

[17] C.Y. Barlow, P. Nielsen y N. Hansen, Acta Mater. 52 (2004) 3.967-3.972.

[18] C.M. Cepeda-Jiménez, P. Hidalgo, M. Pozuelo, O.A. Ruano y F. Carreño, Metall. Mater. Trans. A 41 (2010) 61-72.

[19] A. Rohatgi, D.J. Harach, K.S. Vecchio y K.P. Harvey, Acta Mater. 51 (2003) 2.933-2.957. 